\title{
Beta Streptococcus Group F
}

National Cancer Institute

\section{Source}

National Cancer Institute. Beta Streptococcus Group F. NCI Thesaurus. Code C86192.

A non-taxonomic grouping of beta hemolytic species within the Streptococcus genus

that are assigned to the F group. 